\begin{tabular}{|c|l|}
\hline Title & Smoothing of polycry stall line copper with rough surface by oblique argon-ion irradiation \\
\hline Author(s) & Hino, T.; Nakai, T.; Nishikawa, M.; Hirohata, Y.; Y amauchi, Y. \\
\hline Citation & $\begin{array}{l}\text { Journal of V acuum Science \& Technology B Microelectronics and Nanometer Structures, 24(4), 1918-1921 } \\
\text { https:/doi.org/10.1116/1.2209987 }\end{array}$ \\
\hline Issue Date & $2006-07$ \\
\hline Doc URL & http://hdl.handle.net/2115/14695 \\
\hline Type & article \\
\hline File Information & JV STB2006-24 4.pdf \\
\hline
\end{tabular}

Instructions for use 


\title{
Smoothing of polycrystalline copper with rough surface by oblique argon-ion irradiation
}

\author{
T. Hino \\ Laboratory of Plasma Physics and Engineering, Hokkaido University, Kita-13, Nishi-8, Kita-ku, \\ Sapporo 060-8628, Japan and Science Solutions International Inc., 21-7 Naka-machi, Meguro-ku, \\ Tokyo 153-0065, Japan \\ T. Nakai \\ Laboratory of Plasma Physics and Engineering, Hokkaido University, Kita-13, Nishi-8, Kita-ku, \\ Sapporo 060-8628, Japan \\ M. Nishikawa \\ Science Solutions International Inc., 21-7 Naka-machi, Meguro-ku, Tokyo 153-0065, Japan \\ Y. Hirohata and Y. Yamauchi \\ Laboratory of Plasma Physics and Engineering, Hokkaido University, Kita-13, Nishi-8, Kita-ku, \\ Sapporo 060-8628, Japan and Science Solutions International Inc., 21-7 Naka-machi, Meguro-ku, \\ Tokyo 153-0065, Japan
}

(Received 10 November 2005; accepted 10 May 2006; published 11 July 2006)

\begin{abstract}
Mechanically polished polycrystalline copper was obliquely irradiated by an argon-ion beam with an ion energy of $1 \mathrm{keV}$. The incident angle and fluence of the argon ion were changed to obtain the dependences of incident angle and ion fluence on the surface flatness. After argon-ion irradiation, the surface flatness was examined using atomic force microscopy. Before the irradiation, the sample had a rough surface consisting of groove structures with an average width of $400 \mathrm{~nm}$ and an average elevation of $37 \mathrm{~nm}$. The percentage of the surface area with a height more than $50 \mathrm{~nm}$ was $13 \%$. The incident angle was changed from $0^{\circ}$ (perpendicular injection) to $70^{\circ}$ for the fluence of 1 $\times 10^{18} \mathrm{Ar} / \mathrm{cm}^{2}$. The fluence was changed in the range of $1 \times 10^{17}-1 \times 10^{18} \mathrm{Ar} / \mathrm{cm}^{2}$ for the incident angle of $70^{\circ}$. A smooth surface was obtained when the incident angle was largest, $70^{\circ}$, and the fluence was greater than $5 \times 10^{17} \mathrm{Ar} / \mathrm{cm}^{2}$. The percentage of the surface area with an elevation more than $50 \mathrm{~nm}$ was reduced to $2 \%$, and the number of grooves per unit area was reduced to about half. The reduction of the elevation is mainly due to the selective sputtering for protruding parts, and the reduction of the groove number is mainly due to the redepositing of sputtered particles. The present results show that the oblique ion irradiation is useful for reducing both the elevation and the number of grooves of uneven surfaces. (c) 2006 American Vacuum Society. [DOI: 10.1116/1.2209987]
\end{abstract}

\section{INTRODUCTION}

Flat etching is required for the miniaturization of devices such as large-scale integrated circuits and microelectrical mechanical systems. A wet process such as chemical mechanical polishing (CMP) has been employed as the etching method for copper wiring. ${ }^{1}$ After polishing, an uneven surface remains with roughness from $50-100 \mathrm{~nm}$. Instead of wet etching, dry etching simplifies the etching process because the processing can be performed in only vacuum devices.

The effect of angle of ion incidence on sputtering rate and surface roughness was investigated. ${ }^{2-4}$ Oblique ion etching was done as the dry etching, using helium, argon, or neon to smooth the surfaces of polycrystalline copper, ${ }^{5-7} \mathrm{Cu}(001),{ }^{8}$ $\mathrm{Ag},{ }^{9} \mathrm{SiO}_{2},{ }^{10}$ and $\mathrm{Si}(001) .{ }^{11}$ A copper surface roughened by xenon ion irradiation was smoothed by helium/hydrogen-ion irradiation with an incident angle of $45^{\circ}$ at relatively low ion fluence. ${ }^{8}$ The oblique ion irradiation was conducted on the surface of $\mathrm{Ag}$ at different temperatures, and it was found that elevated temperatures significantly smoothed the surface. ${ }^{9}$ Polycrystalline copper with an initially flat surface was irradiated by helium and argon ions at different incident angles, ${ }^{5,6}$ and we found that the argon-ion irradiation at an incident angle of about $70^{\circ}$ largely reduced the surface roughness caused by blister formation. ${ }^{5}$ The structure of this blister is of ordinal one: i.e., a cavity exists inside the blister. The annealing after the oblique argon-ion irradiation further reduced the surface roughness. ${ }^{7}$ In the oblique argon-ion irradiation for polycrystalline copper, ${ }^{6}$ the surface became rough due to blisters that formed when the ion fluence exceeded about $10^{17} \mathrm{Ar} / \mathrm{cm}^{2}$. However, the surface density and elevation of the blisters were reduced due to the selective sputtering of the protruding parts of the blisters, resulting in a smooth surface. The oblique ion irradiation is useful in reducing the rough surface caused by the blister formation. However, to clarify the advantage of oblique ion irradiation, we must demonstrate that it smoothes the surface of an initially rough structure.

In the present experiment, polycrystalline copper mechanically polished using alumina powder was irradiated using argon-ion irradiation with different incident angles and different ion fluences. After the irradiation, the surface morphology was observed using atomic force microscopy 


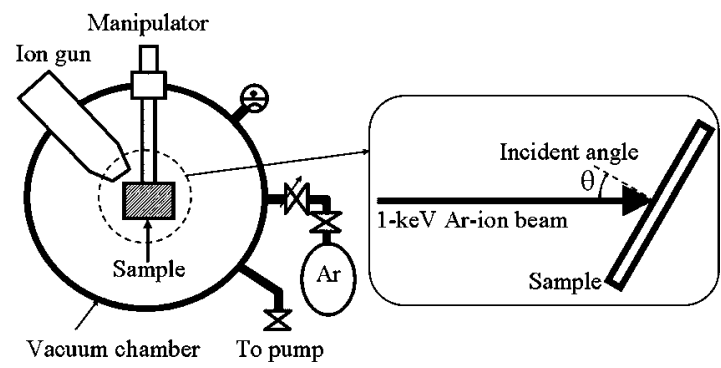

FIG. 1. Schematic illustration of argon-ion irradiation apparatus.

(AFM). The relation of surface flatness with incident angles and ion fluences is discussed.

\section{EXPERIMENTS}

Polycrystalline copper with a purity of 99.99 at. \% made by Nilaco Ltd. was employed as the sample. The $5 \times 15$ $\times 0.1 \mathrm{~mm}^{3}$ sample was mechanically polished using alumina powders with an average size of $3 \mu \mathrm{m}$. The polishing was done on the surface along one direction, and the surface morphology was observed using AFM (SPM-9500, Shimazu Ltd.). The uneven surface due to the polishing had groove structures. After the mechanical polishing, the sample was cleaned in an ultrasonic bath with ethanol.

An ion gun in Auger electron spectroscope (AES) was used for argon-ion irradiation. The sample was placed axially rotated at the sample holder. The incident angle was adjusted by rotating the sample holder. Figure 1 shows the schematic illustration of the oblique ion irradiation apparatus. The energy of argon ions was chosen as $1 \mathrm{keV}$, and the sample temperature was room temperature (RT). The ion flux measured from the sample current was $3 \times 10^{14} \mathrm{Ar} / \mathrm{cm}^{2} \mathrm{~s}$. The incident angles were selected as $0^{\circ}$ (perpendicular), $30^{\circ}$, and $70^{\circ}$ for the ion fluence of $1 \times 10^{18} \mathrm{Ar} / \mathrm{cm}^{2}$. In this experiment, the highest angle was chosen as $70^{\circ}$ because the etching time becomes very long if the angle is taken close to $90^{\circ}$. The ion fluence was chosen from $1 \times 10^{17}$ to 1 $\times 10^{18} \mathrm{Ar} / \mathrm{cm}^{2}$ for the incident angle of $70^{\circ}$.

After the irradiation, the weight loss due to sputtering was measured using a microbalance, and the change of surface morphology was examined using AFM. The surface flatness is expressed as the average elevation of groove $(h)$, average number of grooves per $1 \mu \mathrm{m}\left(N_{g}\right)$, and root mean square (rms) of elevation. The root mean square of elevation is defined by

$$
\mathrm{rms}=\sqrt{\Sigma\left(Y_{i}\langle Y\rangle\right)^{2} / N} \quad(\mathrm{~nm}),
$$

where $Y_{i}$ is the height at the sampling position $i, N$ the sampling number, and $\langle Y\rangle$ the average elevation. Before the irradiation, the region with a height more than $50 \mathrm{~nm}$ existed, although the average elevation was $37 \mathrm{~nm}$. Then, the percentages of surface area with an elevation more than $50 \mathrm{~nm}$ before and after the irradiation were obtained using the AFM software.

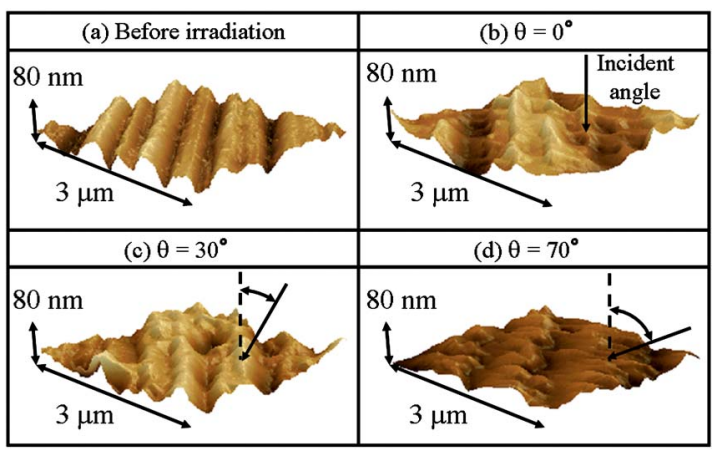

FIG. 2. Surface morphologies after argon-ion irradiation with different incident angles at ion fluence of $1 \times 10^{18} \mathrm{Ar} / \mathrm{cm}^{2}$.

\section{RESULTS}

The surface morphologies and typical elevation profiles after the irradiation with the fluence of $1 \times 10^{18} \mathrm{Ar} / \mathrm{cm}^{2}$ at different incident angles are shown in Fig. 2. Before the irradiation, the average elevation was $37 \mathrm{~nm}$. The width between the grooves was about $400 \mathrm{~nm}$, and the number of grooves per $1 \mu \mathrm{m}$ was 2.5 . After the irradiation with the incident angle of $0^{\circ}$, the surface became rough compared with that before the irradiation. The surface was observed to become smooth with an increase of incident angle. The smoothest surface was observed when the incident angle was $70^{\circ}$. The average elevation was reduced from 37 to $25 \mathrm{~nm}$, and the number of grooves per $1 \mu \mathrm{m}$ was reduced from 2.5 to 1.1. The root mean square of elevation was also reduced from 10.5 to $8.9 \mathrm{~nm}$. The reduced elevation is due to the selective etching of protruding parts, and the reduced groove number is due to the redeposition of sputtered copper atoms. Figure 3 shows the surface morphologies and typical height profiles after the irradiation with an incident angle of $70^{\circ}$ at different ion fluences. When the fluence was 2 $\times 10^{17} \mathrm{Ar} / \mathrm{cm}^{2}$, the surface morphology was roughly the same as that before the irradiation. After irradiation with a fluence of $5 \times 10^{17} \mathrm{Ar} / \mathrm{cm}^{2}$, the surface became significantly smoother. After irradiation with a fluence of 1 $\times 10^{18} \mathrm{Ar} / \mathrm{cm}^{2}$, the surface became smoother than that of 5 $\times 10^{17} \mathrm{Ar} / \mathrm{cm}^{2}$. The root mean square of elevation decreased as ion fluence increased. Table I shows the average eleva-

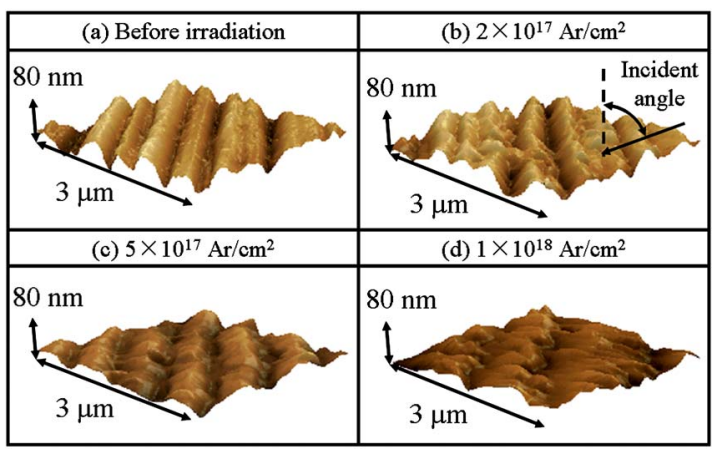

FIG. 3. Surface morphologies after argon-ion irradiation with different ion fluences at incident angle of $70^{\circ}$. 
TABLE I. Average height, average number of grooves per $1 \mu \mathrm{m}$, and root mean square of elevation for different incident angles and different ion fluences.

\begin{tabular}{|l|c|c|c|c|}
\hline & Fluence $\left[\mathrm{Ar} / \mathrm{cm}^{2}\right]$ & $\mathbf{h}[\mathbf{n m}]$ & $\mathrm{N}_{\mathrm{g}}[/ \mu \mathrm{m}]$ & Rms $[\mathbf{n m}]$ \\
\hline (a) Before irradiation & 0 & 37 & 2.5 & 10.5 \\
\hline (b) $\theta=0^{\circ}$ & $1 \times 10^{18}$ & 39 & 1.8 & 15.9 \\
\hline (c) $\theta=30^{\circ}$ & $1 \times 10^{18}$ & 39 & 1.9 & 13.9 \\
\hline \multirow{3}{*}{ (d) $\theta=70^{\circ}$} & $2 \times 10^{17}$ & 35 & 2.4 & 12.1 \\
\cline { 2 - 5 } & $5 \times 10^{17}$ & 27 & 1.8 & 10.4 \\
\cline { 2 - 5 } & $1 \times 10^{18}$ & 25 & 1.1 & 8.9 \\
\hline
\end{tabular}

tions, the average numbers of grooves per $1 \mu \mathrm{m}$, and the root mean squares of elevation for different incident angles and different ion fluences.

In the AFM, the surface morphology was measured by using a cantilever with a tip. The curvature radius of the tip was smaller than $10 \mathrm{~nm}$. A highest resolution length in the $X-Y$ plane is $5-10 \mathrm{~nm}$, and a highest resolution length in the $Z$ axis (height) is much shorter than that in the $X-Y$ plane. The width between grooves was in the range of 400-900 nm, and the elevation was as high as about $100 \mathrm{~nm}$. The elevation was much smaller than the width, and then the tip sidewall does not contact with the groove region in the AFM measurement.

The weight loss after the irradiation of $1 \times 10^{18} \mathrm{Ar} / \mathrm{cm}^{2}$ was about $200 \mu \mathrm{g} / \mathrm{cm}^{2}$. The average etching length was estimated to be $200 \mathrm{~nm}$ for the ion fluence of 1 $\times 10^{18} \mathrm{Ar} / \mathrm{cm}^{2}$. The irradiation with an ion fluence of 5 $\times 10^{17} \mathrm{Ar} / \mathrm{cm}^{2}$ corresponds to the etching length of about $100 \mathrm{~nm}$, which is roughly three times as large as the elevation before the irradiation. For the surface to be smoothed, the length that corresponds to the elevation $h / \sin (\pi / 2-\theta)$ has to be etched. In the case of an incident angle of $70^{\circ}$, this length becomes about $3 h$. Thus, the smoothed surface corresponds to the etching length. For the present rough structure, the surface may be smoother if the incident angle is taken to be more oblique.

Figure 4 shows the percentage of surface area with height more than $50 \mathrm{~nm}$ versus incident angle and the corresponding surface morphology. The percentage of surface area with elevation more than $50 \mathrm{~nm}$ before the irradiation was $12.5 \%$.

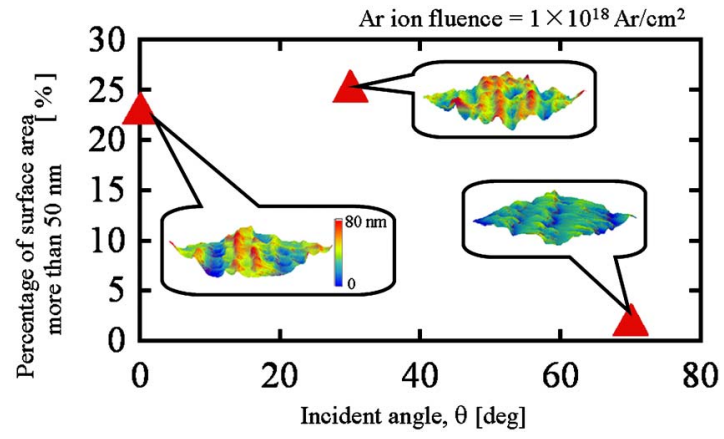

FIG. 4. Percentage of surface area more than $50 \mathrm{~nm}$ vs incident angle at argon-ion fluence of $1 \times 10^{18} \mathrm{Ar} / \mathrm{cm}^{2}$.

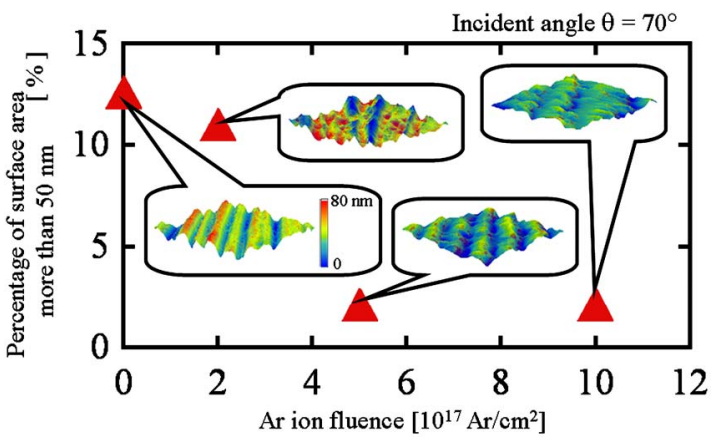

FIG. 5. Percentage of surface area more than $50 \mathrm{~nm}$ vs argon-ion fluence at incident angle of $70^{\circ}$.

After the irradiation with incident angles of $0^{\circ}$ and $30^{\circ}$, the percentage was approximately doubled. This increase is due to nonuniform etching on the surface of the polycrystalline copper consisting of crystal grains with about $80 \mathrm{~nm}$ in diameter and to redeposition of sputtered particles. However, this percentage was significantly reduced to only $2 \%$ after irradiation with an incident angle of $70^{\circ}$ because the protruding parts are preferentially etched. Figure 5 shows the percentage of surface area with elevation more than $50 \mathrm{~nm}$ versus ion fluence and the corresponding surface morphology. After the irradiations with a fluence higher than 5 $\times 10^{17} \mathrm{Ar} / \mathrm{cm}^{2}$, the percentage was significantly reduced. These results indicate that an irradiation with a fluence higher than $5 \times 10^{17} \mathrm{Ar} / \mathrm{cm}^{2}$ and an incident angle larger than $70^{\circ}$ is required to reduce surface roughness in the present experimental conditions.

In the case of the oblique ion irradiation, the cross section of the groove structure gradually changed, as shown in Figs. 6 and 7. The groove structure became random when the incident angle was low, as shown in Fig. 6. In the case of an incident angle of $70^{\circ}$, the cross-sectional view became nonsymmetric with an increase of ion fluence, as shown in Fig. 7. The right region of protruding parts became relatively large when the ion direction was from the right region. This behavior may be interpreted as follows: the sputtering in the left region is relatively dominant compared to that in the right region because the escaping depth of target or copper atom in the left region is relatively shallow. In addition, the

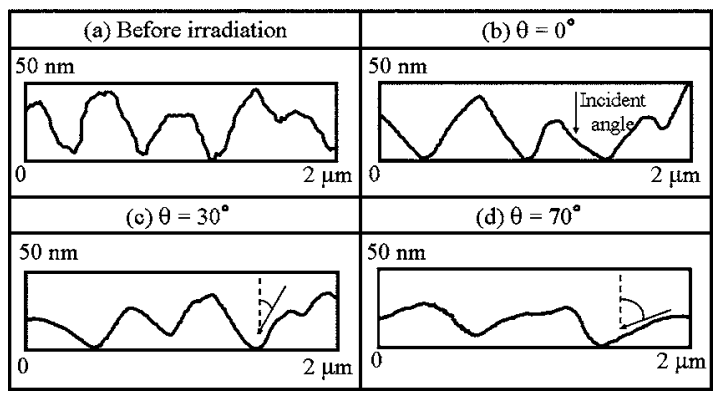

FIG. 6. Cross sections before and after argon-ion irradiation with different incident angles at ion fluence of $1 \times 10^{18} \mathrm{Ar} / \mathrm{cm}^{2}$. 


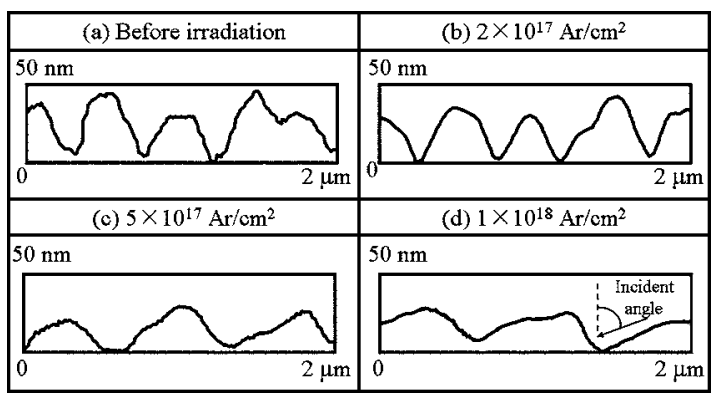

FIG. 7. Cross sections before and after argon-ion irradiation with different ion fluences at incident angle of $70^{\circ}$.

particles sputtered from the left region are deposited in the right region; then, the nonsymmetric cross section is formed.

\section{CONCLUSION}

Argon-ion irradiation was conducted on polycrystalline copper mechanically polished at different ion fluences and different incident angles. The mechanically polished surface had groove structures with an average elevation of $37 \mathrm{~nm}$ and an average number of grooves per $1 \mu \mathrm{m}$ of 2.5. After the irradiation with an ion fluence of $1 \times 10^{18} \mathrm{Ar} / \mathrm{cm}^{2}$ at different incident angles, the average elevation was reduced to $25 \mathrm{~nm}$ and the average number of grooves was reduced to 1.1 when the incident angle was $70^{\circ}$. Because the rough surface was significantly flattened after the irradiation with an incident angle of $70^{\circ}$, the irradiation was conducted at different ion fluences. We observed that the surface became significantly smoother at an ion fluence greater than 5 $\times 10^{17} \mathrm{Ar} / \mathrm{cm}^{2}$. The percentage of surface area with height more than $50 \mathrm{~nm}$ was obtained both before and after the irradiations. The percentage of surface area with height more than $50 \mathrm{~nm}$ was reduced from $12.5 \%$ to $2 \%$ after the irradiation with an ion fluence of $1 \times 10^{18} \mathrm{Ar} / \mathrm{cm}^{2}$ and an incident angle of $70^{\circ}$.

These results suggest that argon-ion irradiation with an ion fluence higher than $5 \times 10^{17} \mathrm{Ar} / \mathrm{cm}^{2}$ at an incident angle of $70^{\circ}$ is very useful for reducing the rough surface of polycrystalline copper. The etching length of this fluence corresponds to the length required for the surface to be smoothed. The average elevation observed in the present irradiation experiments was as low as $25 \mathrm{~nm}$. If the incident angle and the beam intensity are taken higher than the present values, the surface flatness will be further improved. In the oblique ion irradiation, the cross section of the groove structure became nonsymmetric due to nonuniform sputtering and redeposition. The oblique argon-ion irradiation was observed to be effective in smoothing the rough surface, and this technique contributes to the miniaturization of devices such as microelectrical mechanical system and large-scale integrated circuits.

${ }^{1}$ For example, T. Doi, Details Semiconductor CMP Technology (Kogyo Chosakai, Tokyo, 2001) (in Japanese).

${ }^{2}$ H. H. Anderson and H. L. Bay, in Sputtering by Particle Bombardment I, edited by R. Behrisch (Springer, New York, 1981), pp. 145-218.

${ }^{3}$ M. Stepanova and S. K. Dew, Phys. Rev. B 66, 125407 (2002).

${ }^{4}$ G. Garter, J. Phys. D R1, 34 (2001).

${ }^{5}$ Y. Yamauchi, T. Taguchi, Y. Hirohata, T. Hino, and M. Nishikawa, Vacuum 74, 479 (2004).

${ }^{6}$ T. Taguchi, Y. Yamauchi, Y. Hirohata, T. Hino, and M. Nishikawa, Appl. Surf. Sci. 237, 321 (2004).

${ }^{7}$ T. Hino, T. Taguchi, Y. Yamauchi, Y. Hirohata, and M. Nishikawa, J. Vac. Sci. Technol. B 22, 2632 (2004).

${ }^{8}$ M. Ritter, M. Stindtmann, M. Farle, and K. Baberschke, Surf. Sci. 348, 243 (1996).

${ }^{9}$ S. Rusponi, G. Costantini, F. Buatier de Mongeot, C. Boragno, and U. Valbusa, Appl. Phys. Lett. 75, 3318 (1999).

${ }^{10}$ P. Mitra and A. F. Hebard, Appl. Phys. Lett. 86, 063108 (2005).

${ }^{11}$ J. Erlebacher, M. J. Aziz, E. Chason, M. J. Sinclair, and J. A. Floro, Phys. Rev. Lett. 84, 5800 (2000). 